\title{
PHONON DYNAMICS AND LIFETIMES OF AIN AND GAN CRYSTALLITES
}

Leah Bergman*, Dimitri Alexson*, Robert J. Nemanich*, Mitra Dutta**, Michael A.

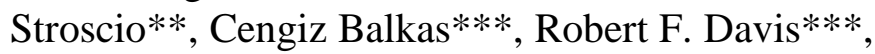

*Department of Physics, North Carolina State University, Raleigh, NC 27695-8202

**U. S. Army Research Office, P. O. Box 12211, Research Triangle Park, NC 27709-2211

***Department of Materials and Science Engineering, North Carolina State University, Raleigh

\section{Cite this article as: MRS Internet J. Nitride Semicond. Res. 4S1, G6.65(1999)}

\section{ABSTRACT}

The quasi-LO and quasi-TO modes of AIN crystallite were investigated. The analysis indicates that the Raman mode behavior concurs with Loudons' model of mode-mixing in wurtzite (WZ) structure crystals which is due to the long-range electrostatic field. Phononlifetimes of GaN and AlN crystallites were studied via Raman lineshape. It was found that the low energy E2 mode lifetime is about an order of magnitude longer than that of the other modes, and that impurities impact significantly the phonon-lifetimes.

\section{INTRODUCTION}

Due to the long range electrostatic field associated with LO phonons, various types of interactions are possible in WZ semiconductors. Among them is the interaction between polar modes belonging to different symmetry groups which results in the creation of new mixedsymmetry phonons, referred to as quasi modes [1-2]. The first part of this paper presents a study of the LO and TO quasi modes in AlN crystallite which originate from the interaction of phonons belonging to the A1 and E1 symmetry groups; the study focuses on the mode-selection rules and mode-frequency calculations as well as on the Raman technique which enables the observation of the quasi-modes [3]. The second part of the paper focuses on Raman analyses of the lifetimes of phonons in GaN and AlN crystallites [4]. The lifetime analyses indicate that the phonon lifetimes in $\mathrm{AlN}$ as well as in GaN fall into two main time regimes: a relatively long time of the low energy E2 mode, which will be referred to as E2(low), and the much shorter times of the high energy E2 mode (referred to as E2(high)) and the E1(TO), A1(TO), and A1(LO) modes. The lifetime shortening due to impurities was also studied: the lifetimes of the Raman modes of an AlN crystallite which contains about two orders of magnitude more Si and $\mathrm{C}$ impurities relative to the concentration of the high quality crystallite were found to be $50 \%$ shorter.

\section{RESULTS AND DISCUSSION}

The theory developed by Loudon formulates that in uniaxial materials the polar phonon characteristics may be affected via two interaction mechanisms: one due to the long range electrostatic field, and the other due to the short range field which exhibits the anisotropy of the vibrational force constants [2]. The phonon dynamics and thus the Raman spectra depend on which of the two mechanism is the dominant interaction.

For the case where the long range electrostatic field is the dominant mechanism, the interaction of the polar phonons with the long range electrostatic field may result in a significant frequency separation between the group of the TO phonons relative to that of the LO phonons. Moreover, the TO phonons belonging to different symmetry are grouped together in a relatively narrow frequency range; the same holds for the LO phonons. One consequence of the dominant electrostatic field interaction is that under certain propagation and polarization conditions, phonons of mixed A1 and E1 symmetry character exist and can be observed in the Raman spectra. These mixed symmetry modes are termed quasi-LO and -TO modes. The frequencies of such quasi modes are predicted by the theory to be between the values of the pure-A1 and the pure-E1 for each of the LO and the TO bands. Alternatively, for the case where the short 
range interatomic forces are dominant, the LO-TO splitting will be small and in this case the TO and LO mode of each symmetry group will occur in a relatively narrow band.

In AIN the E1(TO) and A1(TO) Raman frequencies are grouped together in $\sim 60 \mathrm{~cm}^{-1}$ frequency range, the E1(LO) A1(LO) in $\sim 20 \mathrm{~cm}^{-1}$ range, and the LO-TO group-splitting is $\sim 220$ $\mathrm{cm}^{-1}$. The frequency scheme of AlN thus implies the dominance of the long range electrostatic force interaction. The mode-mixing in AlN may occur if the propagation direction (q-vector) of the quasi-polar phonons, their polarization LO, and one of their two TO's, lie in the plane which spans between the $c$ and the $a_{1}$ (or $a_{2}$ ) crystallographic axes [1]. This plane is referred as to the mixing plane. For example, the pure-A1 phonon has a c-direction polarization while the pureE1 phonon is polarized in the basal plane; thus a quasi-phonon with the q-vector between the c and the $\mathrm{a}_{1}$ axes would exhibit a mixed polarization of A1-E1 symmetry. When the q-vector lies along the crystallographic axes or in the $\mathrm{a}_{1}-\mathrm{a}_{2}$ plane only pure phonons are observed in the spectra.

In this study we observed the AlN quasi-TO and -LO modes by rotating the crystallite relative to the laboratory coordinate system as schematically depicted in Figure 1 . In the figure, the $\mathrm{X}, \mathrm{Y}$, and $\mathrm{Z}$ are the axes of the fixed coordinate laboratory system (where $\mathrm{X}$ is out of the paper plane), while the $c, a_{1}$ and $a_{2}$ are the axes of the rotating coordinate system of the crystal (where $\mathrm{a}_{2}$ is out of the paper plane and is coincided with the $\mathrm{X}$-axis), and $\mathrm{N}$ is the normal to the crystallite surface (and is coincided with the $\mathrm{a}_{1}$-axis). The AlN crystallite was rotated through an angle $\theta$ about the $\mathrm{X}$-axis such that the quasi-phonon propagation direction, the q-vector $(\mathbf{q})$, as well as its LO and one of its two TO components lay in the mixing $\mathrm{c}-\mathrm{a}_{1}$ plane (the other TO is along the $\mathrm{X}$-axis). The micro-Raman spectra was acquired in a back scattering geometry from the $\mathrm{Y}$-axis for various values of $\theta$.

In order to investigate the polarization conditions upon which the quasi modes appear in the spectra, the Raman tensors, $\mathrm{R}_{\theta}$, of the rotating crystal (relative to a fixed $\mathrm{X}, \mathrm{Y}, \mathrm{Z}$ laboratory coordinate system) are calculated via the rotational transformation equation [5]: $R_{\theta}=T_{x} R T_{x}^{-1}$ where $\mathrm{T}_{\mathrm{x}}$ is the rotation matrix about the $\mathrm{X}$-axis. The Raman tensors of the $\mathrm{WZ}$ structure take the form:

$$
\begin{aligned}
& A 1_{c}(\theta)=\left(\begin{array}{ccc}
h & 0 & 0 \\
0 & h \cos ^{2} \theta+b \sin ^{2} \theta & b \sin \theta \cos \theta-h \sin \theta \cos \theta \\
0 & b \sin \theta \cos \theta-h \sin \theta \cos \theta & h \sin ^{2} \theta+b \cos ^{2} \theta
\end{array}\right) \\
& E 1_{x}(\theta)=\left(\begin{array}{ccc}
0 & d \sin \theta & d \cos \theta \\
d \sin \theta & 0 & 0 \\
d \cos \theta & 0 & 0
\end{array}\right) \\
& E 2(\theta)=\left(\begin{array}{ccc}
0 & f \cos \theta & -f \sin \theta \\
f \cos \theta & 0 & 0 \\
-f \sin \theta & 0 & 0
\end{array}\right) \quad E 2(\theta)=\left(\begin{array}{ccc}
f & 0 & 0 \\
0 & -f \cos ^{2} \theta & f \cos \theta \sin \theta \\
0 & f \cos \theta \sin \theta & -f \sin ^{2} \theta
\end{array}\right) \\
& \begin{aligned}
E 1_{\mathrm{a}_{1}}(\theta) & =\left(\begin{array}{ccc}
0 & 0 & 0 \\
0 & 2 \mathrm{~d} \sin \theta \cos \theta & \mathrm{d}\left(\cos ^{2} \theta-\sin ^{2} \theta\right) \\
0 & \mathrm{~d}\left(\cos ^{2} \theta-\sin ^{2} \theta\right) & -2 \mathrm{~d} \sin \theta \cos \theta
\end{array}\right) \\
E 2(\theta) & =\left(\begin{array}{ccc}
f & 0 & 0 \\
0 & -f \cos ^{2} \theta & f \cos \theta \sin \theta \\
0 & f \cos \theta \sin \theta & -f \sin ^{2} \theta
\end{array}\right)
\end{aligned}
\end{aligned}
$$

The tensors describe the in-crystal projections, due to the rotation, of the incident and scattered polarization of the light. Moreover, for each polar mode the direction of the polarization is indicated in the subscripts: A1 is polarized along the $\mathrm{c}$ axis while $\mathrm{E} 1$ is polarized along $\mathrm{a}_{1}$ and $\mathrm{a}_{2}$, the latter which coincides with the $\mathrm{X}$-axis $\left(\mathrm{E} 1_{\mathrm{X}}\right)$. The polarization direction of the polar modes, like the Raman tensors, can be expressed in terms of the laboratory coordinate system; however, it is not necessary for the present study. For $\theta=0$ the crystal coordinate system coincides with that of the laboratory $\left(a_{1}=Y\right.$ and $c=Z$, see Fig. 1), and Eq. 1 takes the usual form [1-2].

For the first set of experiments we chose the Raman setup such that the incident light is polarized along the $\mathrm{X}$-axis and the scattered light has both $\mathrm{X}$ and $\mathrm{Z}$ polarization directions, expressed in Porto notation [6] as: $\mathrm{Y}\left(\mathrm{X}_{\mathrm{Z}}^{\mathrm{X}}\right) \overline{\mathrm{Y}}$. This choice of polarization implies that the $\mathrm{XX}$ and the XZ components of each of the Raman tensors will contribute a Raman signal provided 
they are not zero. Figure 2 presents the Raman spectra for this setup for rotations: $\theta=0^{\circ}, 35^{\circ}$, and $70^{\circ}$. Inspection of Eq. 1 indicates that the E2 mode should appear in the spectra since the $\mathrm{XX}$ and the XZ components of its tensors are not zero; our spectra display the E2 mode at 655 $\mathrm{cm}^{-1}$. This mode is non-polar and as such no change in frequency is expected as a function of the rotation. The $\mathrm{XZ}$ component of the $\mathrm{E} 1_{\mathrm{x}}$ is non-zero, and although the q-vector of the mode lies in the a-c mixing plane its polarization is not: it is in the X-direction (TO component) and thus no symmetry mixing is expected. This analysis indicates that the spectra should exhibit the pure-E1(TO) with no change of frequency upon rotation. The pure-E1(TO) in our spectra is at $669 \mathrm{~cm}^{-1}$ and is independent of rotation.

Next we investigated the A1 mode which contributes a signal to the Raman spectra via the non-zero value of its XX tensor component. The pure-A1 mode has solely c-polarization; however, upon rotating the crystal quasi modes are created for which their TO and LO components have a c-polarization component of A1 symmetry as well as an a-polarization component of E1 symmetry. This situation is schematically depicted in the inset to Figure 1 . In the figure, the TO component of the quasi-mode lies in the mixing plane and is composed of A1 and $\mathrm{E} 1$ polarization symmetry components; the quasi-TO mode will have mostly A1 symmetry for q-vectors near the a-axis and mostly E1 symmetry for q-vectors near the c-axis. The frequency of the quasi-TO, according to Loudons' theory, should be between the frequency of the pure-A1(TO) mode and that of the pure-E1(TO) mode [2]. As can be seen in Figure 2, the frequency of the A1(TO) mode shifts from its pure value at $608 \mathrm{~cm}^{-1}$ towards that of the E1(TO) mode as a function of the rotation angle: for $\theta=35^{\circ}$ the peak of the quasi-TO is at $614 \mathrm{~cm}^{-1}$ and for $\theta=70^{\circ}$ is at $620 \mathrm{~cm}^{-1}$. The spectrum of $\theta=70^{\circ}$ exhibits a small peak of the pure-A1(TO) and is attributed to an internal reflection.

The frequencies of the quasi-TO can be calculated via the relation [1-2]

$$
\omega_{\mathrm{Q}(\mathrm{TO})}^{2}=\omega_{\mathrm{E} 1(\mathrm{TO})}^{2} \cos ^{2}(90-\beta)+\omega_{\mathrm{A} 1(\mathrm{TO})}^{2} \sin ^{2}(90-\beta)
$$

where $\beta$ (see Fig. 1) is the propagation angle of the q-vector of the quasi-phonon $(\mathbf{q})$ in the crystallite, and is related to the angle of rotation, $\theta$, via Snell's law and the momentumconservation law of the Raman scattering effect as is discussed next. The conservation law states that in the scattering medium $\mathbf{q}=\mathbf{k}_{\mathrm{I}}-\mathbf{k}_{\mathrm{S}}$ where $\mathbf{k}_{\mathrm{I}}$ and $\mathbf{k}_{\mathrm{S}}$ are wavevectors of the incident and scattered photons respectively [2]. Thus, for our backscattering geometry the q-vector lies along the same path as that of the incident and scattered photons. To find the angle, $\beta$, of that path the index of refraction has to be taken into account via Snell's law: $n_{1} \sin \theta=n_{2} \sin \beta$, where for air $n_{1}=1$ and for $A l N n_{2}=2.2$ which is the accepted value in the visible range and of negligible anisotropy [7]. Our calculations yield a value of $\beta=15^{\circ}$ for the rotation $\theta=35^{\circ}$, and from Eq. 2, $\omega_{\mathrm{Q}(\mathrm{TO})}=612 \mathrm{~cm}^{-1}$, a value which concurs with our experimental finding of $614 \mathrm{~cm}^{-1}$. For the $70^{\circ}$ rotation, $\beta=25^{\circ}$ and $\omega_{\mathrm{Q}(\mathrm{Tо})}=619 \mathrm{~cm}^{-1}$, a value which is similar to the experimental result of 620 $\mathrm{cm}^{-1}$.

Lastly we investigated the quasi-LO Raman mode. In order to observe this mode the incoming polarization of the light was set at the Z-direction such that the Raman geometry was $\mathrm{Y}\left(\begin{array}{l}\mathrm{Z} \\ \mathrm{Z}\end{array}\right) \overline{\mathrm{Y}}$. This Raman geometry enables the observation of the quasi-LO modes since the ZZ component of the $\mathrm{E}_{\mathrm{a}}$ Raman tensor is non-zero for $\theta>0$ as is indicated by Eq. 1. In this configuration the q-vector of the phonon as well as its polarization directions lie in the mixing plane. Figure 3 presents the Raman spectra of the quasi-LO mode for $\theta=15^{\circ}, 35^{\circ}$, and $70^{\circ}$, and the pure A1(LO) mode at $\theta=90^{\circ}$; the Raman peak positions are at $913 \mathrm{~cm}^{-1}, 912 \mathrm{~cm}^{-1}, 908 \mathrm{~cm}^{-1}$, and $890 \mathrm{~cm}^{-1}$ respectively. Taking into account the index of refraction and utilizing the equation for the quasi-LO frequencies [1-2], 


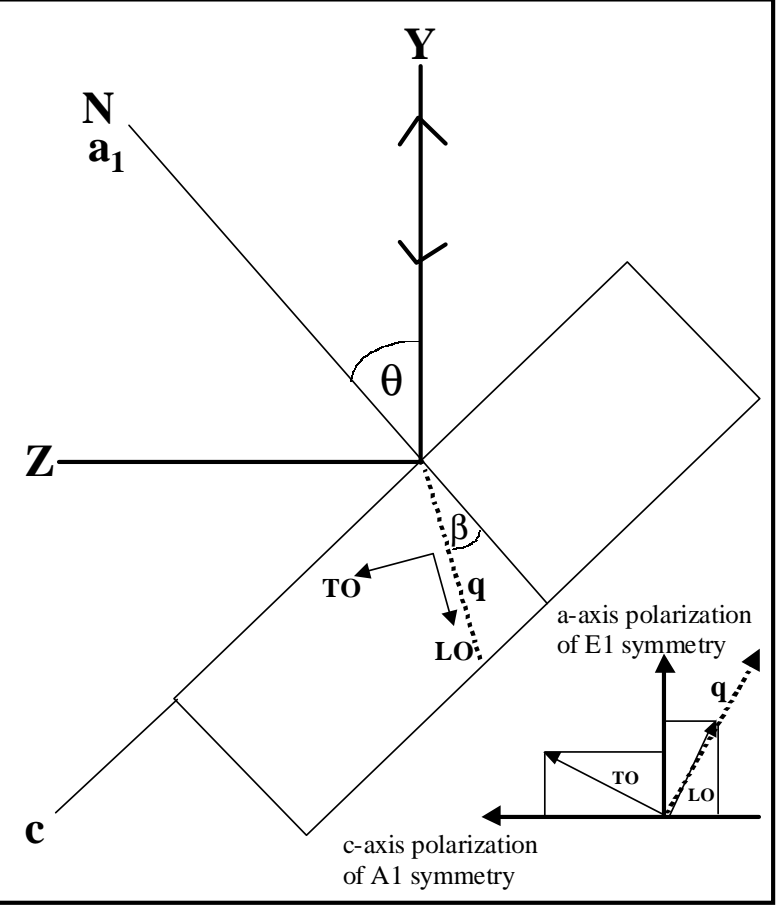

Fig. 1. The experimental setup. The laboratory system: X (out of the paper), Y, and Z. The rotating crystal system: $\mathrm{c}, \mathrm{a}_{1} . \mathrm{N}$ is the normal and $\theta$ is the rotation angle about the $X$-axis, $\beta$ is the angle of refraction and defines phonon propagation. The dashed line represents the phonon q-vector the arrows are its LO and TO components. The Raman spectra was acquired in a back scattering geometry from the $\mathrm{Y}$ axis.

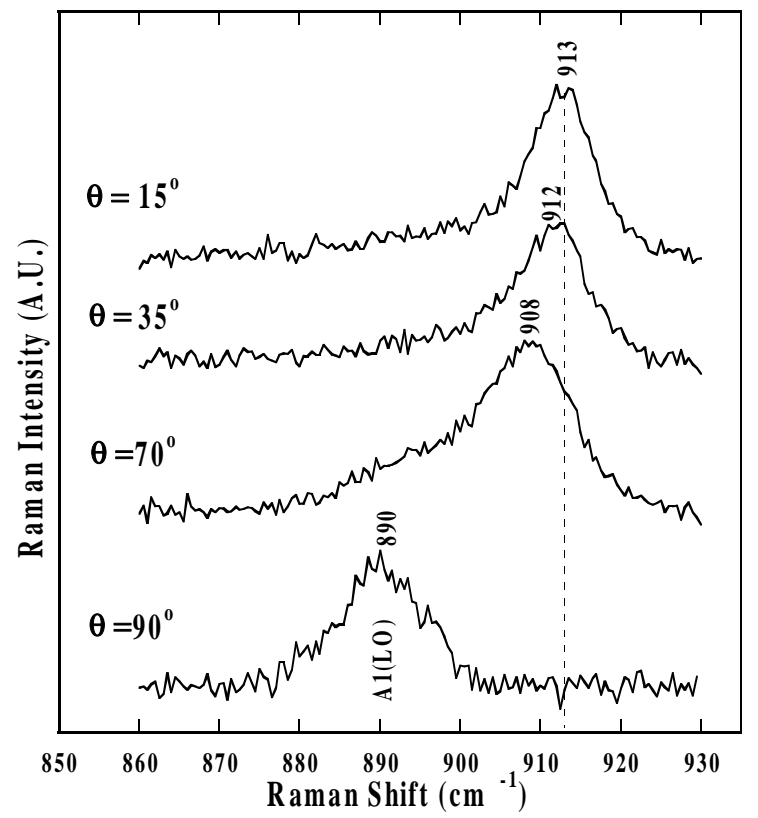

Fig. 3. The quasi-LO modes: 913, 912, and $908 \mathrm{~cm}^{-1}$. The pure A1(LO) is at 890 $\mathrm{cm}^{-1}$.

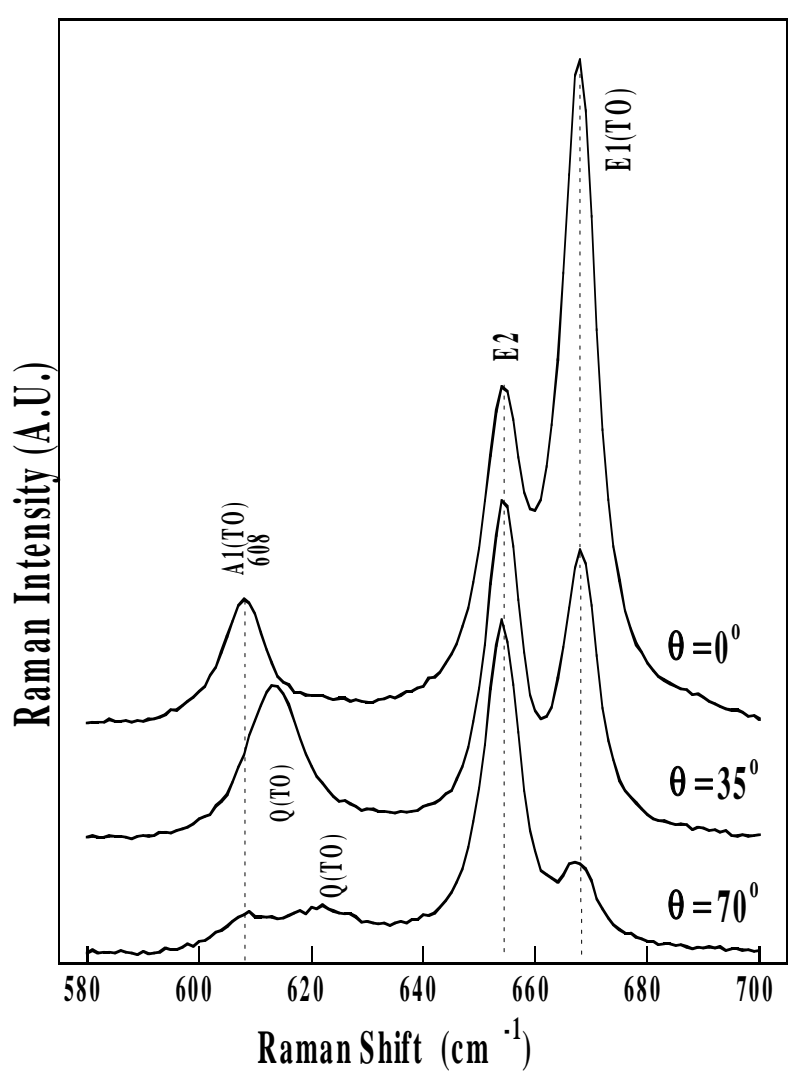

Fig. 2. The quasi-TO modes: 620, and $614 \mathrm{~cm}^{-1}$. the pure A1(TO) is at $608 \mathrm{~cm}^{-1}$.

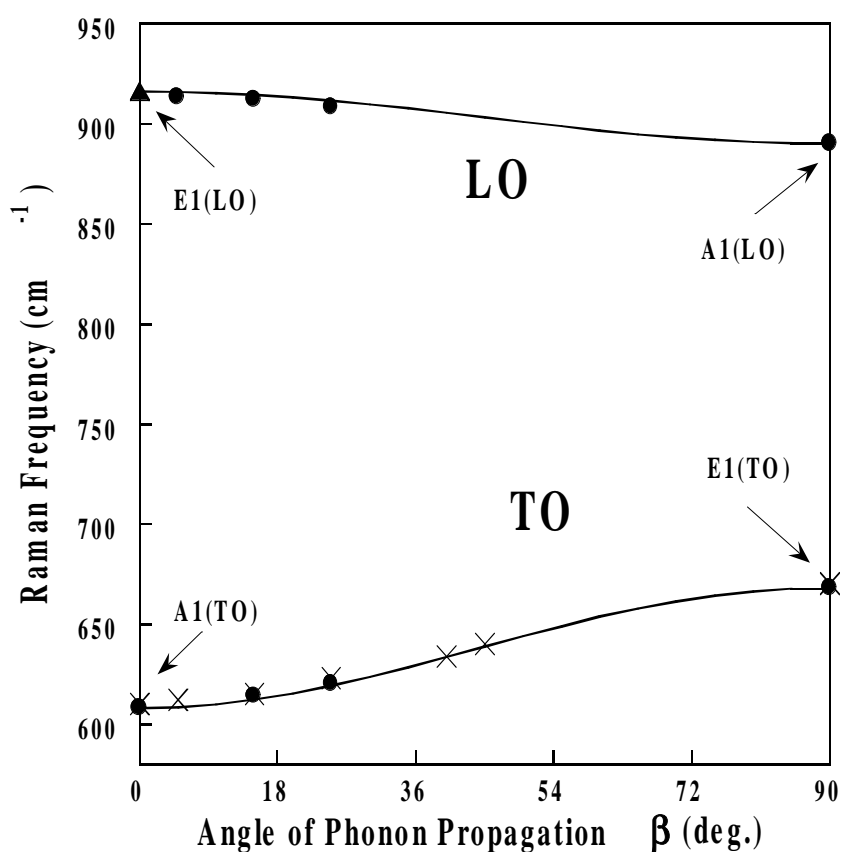

Fig. 4. The quasi-mode frequency as a function of the phonon-propagation direction. The lines represent Loudons' model, the dots our data, and the x's are from [9]. 


$$
\omega_{\mathrm{Q}(\mathrm{LO})}^{2}=\omega_{\mathrm{A} 1(\mathrm{LO})}^{2} \cos ^{2}(90-\beta)+\omega_{\mathrm{E} 1(\mathrm{LO})}^{2} \sin ^{2}(90-\beta)
$$

where $\omega_{\mathrm{ad}(\mathrm{LO})}$ is $890 \mathrm{~cm}^{-1}$ and $\omega_{\mathrm{E} 1(\mathrm{LO})}$ is $916 \mathrm{~cm}^{-1}$ [8], we found the calculated quasi frequencies to be $915 \mathrm{~cm}^{-1}, 914 \mathrm{~cm}^{-1}$, and $911 \mathrm{~cm}^{-1}$, which agree well with the above experimental values.

Figure 4 summarizes our findings. In the figure the lines denote the TO and LO quasifrequencies calculated utilizing Loudons' model (Eqs. 2 and 3), and the dots represent our experimental values for the modes. The additional data points of the quasi-TO modes, represented by X's, are from Filippidis et al who were the first to observe the quasi-TO modes in AlN [9]; our results are in agreement with theirs. Moreover, as depicted in the figure, our study of the quasi-TO as well as the quasi-LO concur with Loudons' model, implying the presence of a long range electrostatic field which is active in the coupling of modes belonging to the different symmetry groups.

\section{PHONON-LIFETIMES}

In order to obtain the phonon-lifetime via the Raman spectral linewidth, the linewidth has to be corrected for the contribution of the instrumental bandpass broadening. In order to do so, the Raman spectra were acquired at successive slit widths ranging from $400 \mu \mathrm{m}$ down to 100 $\mu \mathrm{m}$; the measured linewidth-values, $\mathrm{W}_{\mathrm{M}}$, were plotted as a function of the slit width, $\mathrm{W}_{\mathrm{s}}$, and extrapolated to the zero slit-value, $\mathrm{W}_{\mathrm{p}}$, via the relation:

$$
\mathrm{W}_{\mathrm{M}}=\sqrt{\mathrm{W}_{\mathrm{P}}^{2}+\left(\mathrm{W}_{\mathrm{S}} * 9.2 * 10^{-3}\right)^{2}}
$$

The second term in Eq. 4 is the instrumental bandpass, i.e., the slit width multiplied by the monochromator linear dispersion, $9.2 * 10^{-3} \mathrm{~cm}^{-1} / \mu \mathrm{m}$. This method yields the actual phonon linewidth, $\mathrm{W}_{\mathrm{P}}$, from which the phonon lifetime can be calculated.

In general, two main mechanisms control the phonon lifetimes: the phonon anharmonic interactions in which a phonon decays into other phonons, and the phonon scattering at impurity or defect centers [10-11]. The energy conservation law of the anharmonic decay requires that the energy of the decay phonon be equal to the sum of the energies of the created phonons. In the following we present a study of phonon-lifetime in two types of AlN crystallites, referred to as blue and transparent, which differ mainly in their impurity contents: the blue crystallite contains about two orders of magnitude more Si and C impurities then the transparent AlN [12].

In order to calculate the phonons lifetime via the Raman linewidths, the linewidths were plotted as a function of the slit width and a curve fit was obtained via Eq. 4. The results are presented in Fig. 5: the dots represent the data taken from the transparent AlN while the squares data from the blue crystallite. It is evident from the figure that the linewidth of all the Raman modes are strongly correlated with the impurity concentration. The values of the Raman linewidths at the zero slit width may be used to evaluate the phonons lifetime, $\tau$, via the energytime uncertainty relation: $\Delta \mathrm{E} / \hbar=1 / \tau$. Where $\Delta \mathrm{E}$ is the Raman linewidth in units of $\mathrm{cm}^{-1}$, and $\hbar$ $=5.3 * 10^{-12} \mathrm{~cm}^{-1}$-sec. Table I summarizes the results: for each sample the zero slit linewidth is presented along with the calculated lifetime of the Raman modes. Two main conclusions can be drawn from the above results: the lifetimes are correlated with the impurity concentration, and the E2(low) mode of both samples has a significantly longer lifetime than that of the E2(high), E1(TO), A1(TO), and A1(LO) modes. The lifetimes of an high quality GaN crystallite were analyzed as well; the lifetimes computed via the above described method are summarized in Table I. Similar to the AlN lifetimes, the E2(low) mode of the GaN has a significantly longer lifetime than that of the E2(high), E1(TO), and A1(TO) modes. The A1(LO) was not observed in the spectra and its absence is attributed to the plasmon damping. The relatively long lifetime of the E2(low) mode is attributed to its small anharmonic decay probability. Unlike the other modes, the energy of the E2(low) mode lies at the low energy regime of the wurtzite dispersion curve, and only the acoustical phonons are available as a channel of decay [13]. Inspection of the dispersion curves reveals that in order for the energy conservation to hold the E2(low) mode is allowed to decay into acoustical modes of very low density of state. This implies the small probability of decay and thus the long lifetime; a model calculation will be presented at a later date. 
Table I. Linewidth $\Gamma\left(\mathrm{cm}^{-1}\right)$ and lifetime $\tau$ (ps)

\begin{tabular}{|c|c|c|c|c|c|c|}
\hline & \multicolumn{2}{|c|}{$\begin{array}{r}\text { AlN } \\
\text { Transp. }\end{array}$} & \multicolumn{2}{|c|}{$\begin{array}{r}\text { AlN } \\
\text { Blue }\end{array}$} & \multicolumn{2}{|c|}{ GaN } \\
\hline Mode & $\Gamma$ & $\tau$ & $\Gamma$ & $\tau$ & $\Gamma$ & $\tau$ \\
\hline E2(low) & 1.2 & 4.4 & 2.6 & 2.0 & 0.5 & 10.1 \\
\hline E2(high) & 6.4 & 0.83 & 12.4 & 0.43 & 3.9 & 1.4 \\
\hline E1(TO) & 5.8 & 0.91 & 10.0 & 0.53 & 5.6 & 0.95 \\
\hline A1(TO) & 7.0 & 0.76 & 14.4 & 0.37 & 11.6 & 0.46 \\
\hline A1(LO) & 11.9 & 0.45 & 18.9 & 0.28 & & \\
\hline
\end{tabular}

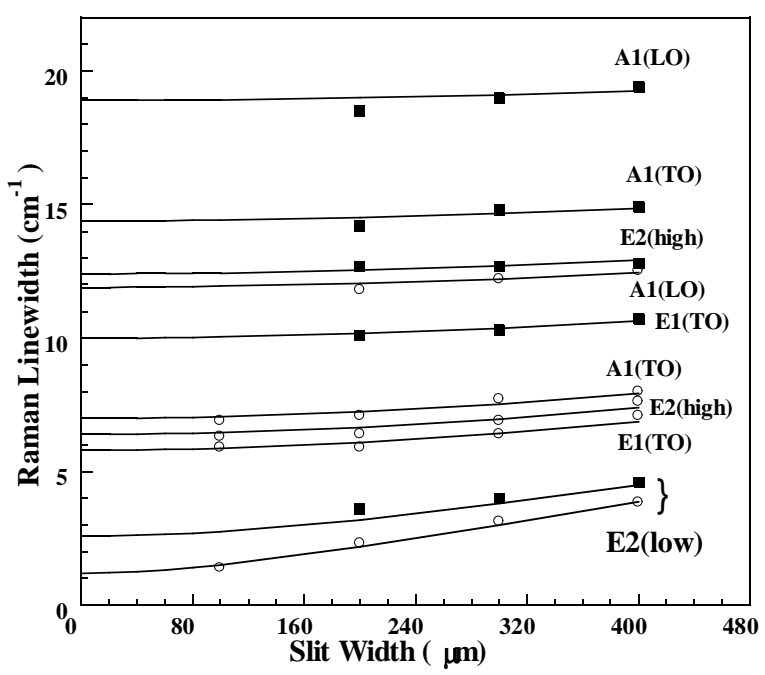

Fig. 5. The linewidth as a function of slitwidth. Data from transparent AlN: circles; blue AlN: squares.

\section{CONCLUSIONS}

The analyses indicate that the Raman mode behavior in AlN concurs with Loudons' model of mode-mixing in WZ structure which is due to the dominance of the long-range electrostatic field. Phonon-lifetimes of $\mathrm{GaN}$ and AlN crystallites were studied via Raman lineshape. It was established that the E2(low) mode lifetime is about an order of magnitude longer than that of the other modes, and that impurities significantly shorten the phononlifetimes.

\section{ACKNOWLEDGEMENTS}

The author acknowledges the National Research Council and the Army Research Office for supporting this research.

\section{REFERENCES}

1. C.A. Arguello, D.L. Rousseau, and S.P.S. Porto, Phys. Rev. 181, 1351 (1969).

2. R. Loudon, Advan. Phys. 13, 423 (1964).

3. L. Bergman, M. Dutta, R.J. Nemanich, and R.F. Davis, To appear in J. Appl. Phys. (1999).

4. L. Bergman, M. Dutta, M.A. Stroscio, R.J. Nemanich, and R.F. Davis, To appear in Phys. Rev.B. (1999).

5. D. A. Long, Raman Spectroscopy, (McGraw-Hill, New York, 1977).

6. S.P.S Porto, in Light Scattering Spectra of Solids, (G.B. Wright, ed. Springer-Verlag, Berlin, 1969).

7. S. Loughin, and R. H. French, Properties of Group III Nitrides, p. 175. Ed. J. H. Edgar, (INSPEC, London, 1994).

8. L.E. McNeil, M. Grimsditch, and R.H. French, J. Am. Ceram. Soc. 76, 1132 (1993).

9. L. Filippidis, H. Siegle, A. Hoffmann, C. Thomsen, K. Karch, and F. Bechstedt, Phys. Stat. Sol. B198, 621 (1996).

10. P.G. Klemens, Phys. Rev. 148, 845 (1966).

11. K.T. Tsen, D.K. Ferry, A. Botchkarev, B. Sverdlov, A. Salvador, and H. Morkoc, Appl. Phys. Lett.

72, 2132 (1998).

12. C.M. Balkas, Z. Sitar, T. Zheleva, L. Bergman, R.J. Nemanich, and R.F. Davis. J. Crys. Growth 179, 363 (1997).

13. J.C. Nipko, and C.K. Loong, Phys. Rev. B57, 10550 (1998). 